\title{
Detección de Rickettsia spp. en ectoparásitos de animales domésticos y silvestres de la Reserva Natural Privada Cerro Chucantí y comunidades aledañas, Panamá, 2007-2010
}

\author{
Sergio Bermúdez¹, Roberto Miranda¹, Yamitzel Zaldívar², Publio González², Guido Berguido³, \\ Diomedes Trejos ${ }^{4}$, Juan M. Pascale ${ }^{2}$, Marcelo Labruna ${ }^{5}$ \\ 1 Departamento de Entomología Médica, Instituto Conmemorativo Gorgas de Estudios de la Salud, Panamá, \\ Panamá \\ 2 Departamento de Genómica y Proteómica, Instituto Conmemorativo Gorgas de Estudios de la Salud, Panamá, \\ Panamá \\ ${ }^{3}$ Reserva Natural Privada Cerro Chucantí, Panamá \\ 4 Instituto de Medicina Legal, Panamá, Panamá \\ ${ }^{5}$ Departamento de Medicina Veterinaria Preventiva e Saúde Animal, Universidad de Sao Paulo, Brasil
}

Introducción. Los ectoparásitos son los principales vectores de rickettsiosis. En Panamá se tienen escasos datos sobre los artrópodos que pudieran considerarse vectores o reservorios.

Objetivos. Presentar datos sobre la presencia de Rickettsia spp. en ectoparásitos de fauna silvestre y animales domésticos en la Reserva Natural Privada Cerro Chucantí y poblados vecinos.

Materiales y métodos. Se revisaron 9 personas, 95 mamíferos domésticos y 48 silvestres. Los animales domésticos se examinaron con anuencia del propietario, mientras que la fauna silvestre se capturó con trampas Sherman y Tomahawk. Se extrajeron 21 especies de ectoparásitos: pulgas, piojos, garrapatas y otros ácaros, los cuales se preservaron en etanol al $95 \%$. Se extrajo material genético de garrapatas y pulgas para ser analizado por técnicas moleculares en la detección de Rickettsia spp.

Resultados. Se practicaron 425 reacciones de PCR, de las cuales, 270 resultaron negativas y 155 positivas. De las positivas, 86 amplificaron para el gen gltA (55\% de las positivas); de estos también amplificaron 41 (26\%) para ompA. Se encontró material genético de Rickettsia amblyommii, en garrapatas de caballos (Amblyomma cajennense, Dermacentor nitens), de perros (Rhipicephalus sanguineus) y ninfas de Amblyomma recolectadas en el bosque. Además, se detectó ADN de $R$. felis en pulgas Ctenocephalides felis de perros.

Conclusiones. Se pudo detectar la presencia de $R$. amblyommii y $R$. felis en garrapatas y pulgas de animales domésticos de los poblados cercanos a Cerro Chucantí, aun cuando no se pudo encontrar material genético de Rickettsia en ectoparásitos de la fauna silvestre.

Palabras clave: Rickettsia, vectores de enfermedades, animales domésticos, animales salvajes, biología molecular, Panamá

Detection of Rickettsia in ectoparasites of wild and domestic mammals from the Cerro Chucanti private reserve and from neighboring towns, Panamá, 2007-2010

Introduction. Ectoparasites are the main vectors of rickettsiosis. In Panama, however, limited data are available concerning the arthropod species that serve as vectors or reservoirs.

Objectives. Data are presented concerning the presence of Rickettsia in ectoparasites of wildlife and domestic animals in the Cerro Chucantí private nature reserve and in neighboring villages.

Materials and methods. Nine humans, 95 domestic mammals and 48 wild mammals were examined. Twenty-one species of ectoparasites were obtained, including fleas, lice, ticks and mites. These were preserved in $95 \%$ ethanol. Later, the DNA was extracted from the ticks and fleas and analyzed by molecular techniques to detect presence of Rickettsia.

Results. Of a total of 425 PCR reactions, 270 were positive for Rickettsia and 155 negative. Among the positive samples, 86 PCR amplified for the gltA gene ( $55 \%$ of positives) and 41 of these also amplified

\footnotetext{
Contribución de los autores:

Sergio Bermúdez coordinó las actividades del proyecto, además de identificar los ectoparásitos, analizar los resultados y escribir el manuscrito.

Roberto Miranda se encargó de las giras de campo.

Yamitzel Zaldívar se encargó de las pruebas de laboratorio.

Guido Berguido y Publio González identificaron los mamíferos silvestres.

Diomedes Trejos, Juan Pascale y Marcelo Labruna, contribuyeron con la interpretación de los análisis moleculares y secuencias.
} 
the ompA gene. DNA of Rickettsia amblyommii was found in horses ticks (Amblyomma cajennense, Dermacentor nitens), dogs ticks (Rhipicephalus sanguineus) and free living nymphs in the forest. Additionally, DNA of $R$. felis was found in fleas from dogs Ctenocephalides felis.

Conclusions. The presence of $R$. amblyommii and $R$. felis was detected in ticks and fleas of domestic animals in villages near Cerro Chucanti; however no Rickettsia DNA was found in ectoparasites of nondomestic wildlife.

Key words: Rickettsia, disease vectors; animals, domestic; animals, wild; domestic and wild animals, molecular biology, Panamá.

Se sabe que la caza tiene un impacto directo en la pérdida de hábitat y biodiversidad, además del desequilibrio ecológico causado por otras actividades humanas como la tala de árboles, la minería y la agricultura, entre otras. Este desequilibrio incide sobre las relaciones ecológicas que, en el caso de las relaciones entre parásitos y huéspedes, pudieran favorecer el establecimiento de nuevos nichos parasitarios (1). Como ejemplo de estas rupturas se tiene el hallazgo de ectoparásitos de fauna silvestre en animales domésticos e incluso humanos $(1,2)$. Paralelamente, esto ha provocado la aparición de zoonosis relacionadas con ectoparásitos en áreas rurales o agrícolas colindantes con zonas naturales, lo cual hace necesarios estudios que involucren a los posibles vectores o reservorios.

Históricamente, las rickettsiosis están dentro de las zoonosis más conocidas, las cuales son causadas por bacterias Gram negativas que se multiplican obligatoriamente dentro de células eucariotas (3). Estas bacterias se relacionan estrechamente con diferentes grupos de invertebrados; sin embargo, algunas especies asociadas con ectoparásitos se consideran patógenas para vertebrados. Según Fournier y Raoult (3), las rickettsiosis se divide en tres grupos: el tifus, asociado principalmente a piojos y pulgas; la de las fiebres manchadas, asociadas principalmente a garrapatas, y una intermedia relacionada con ácaros y otros artrópodos.

Los síntomas de las rickettsiosis son similares al de otras enfermedades febriles, lo cual hace que sean confundidas y subestimadas. En casos no tratados, la tasa de mortalidad puede variar entre 10 y $80 \%$ (4). Desde inicios del siglo XX hasta inicios del presente, en los países latinoamericanos solo se mantenían registros de rickettsiosis humanas por

Correspondencia:

Sergio E. Bermúdez, Departamento de Entomología Médica, Instituto Conmemorativo Gorgas de Estudios de la Salud, Panamá, Panamá.

Teéfono: (507) 5274894

bermudezsec@gmail.com

Recibido: 30/08/11; aceptado:29/12/11
Rickettsia typhi (grupo tifus) y R. rickettsii (grupo de las fiebres manchadas) (5).

En Panamá, desde mediados del siglo pasado se tienen reportes de casos humanos de tifus "murino" (6) y de fiebre manchada por $R$. rickettsii (7). Recientemente, se han registrado en Panamá nuevos brotes de fiebre manchada por $R$. rickettsii (4) y, además, existen reportes ecológicos de ectoparásitos infectados por $R$. rickettsii (8), Rickettsia spp. (9), R. amblyommii (10-12) y $R$. felis (12).

El objetivo de este trabajo es incrementar el conocimiento sobre la presencia de Rickettsia spp. en garrapatas y pulgas recolectadas de animales domésticos y silvestres de una zona no endémica de rickettsiosis en Panamá.

\section{Materiales y métodos}

\section{Zonas de muestreo}

La Reserva Natural Privada Cerro Chucantí se localiza en el cerro homónimo, el cual pertenece a la sección oriental de la Serranía del Majé, provincia de Darién (figura 1). Esta reserva está constituida por bosques primarios del tipo premontano y nuboso, en altitudes entre 600 y 1.480 msnm, y cuenta con una extensión de $240 \mathrm{~km}^{2}$ (13). Fuera de ésta, se encuentran terrenos para pastoreo y alrededor de 15 pueblos, los cuales se dedican principalmente a la agricultura.

De abril 2007 a marzo 2010, se hicieron seis giras de trabajo durante las cuales se revisaron al azar animales domésticos de 15 pueblos cercanos a la Reserva. Igualmente, se capturaron garrapatas en fases no parasíticas, por medio de muestreos con tela blanca $(45 \times 45 \mathrm{~cm})$ en intersecciones de $20 \mathrm{~m}$ lineales dentro del bosque y cubriendo parcelas de $100 \mathrm{~m}^{2}$ dentro de los pastizales contiguos a la reserva.

Para la extracción de ectoparásitos de fauna silvestre dentro de la Reserva, se utilizaron 250 trampas Sherman y 50 Tomahawk, las cuales se colocaron en intersecciones lineales que cubrían altitudes de 600, 800, 1.000 y 1.400 msnm. Las 


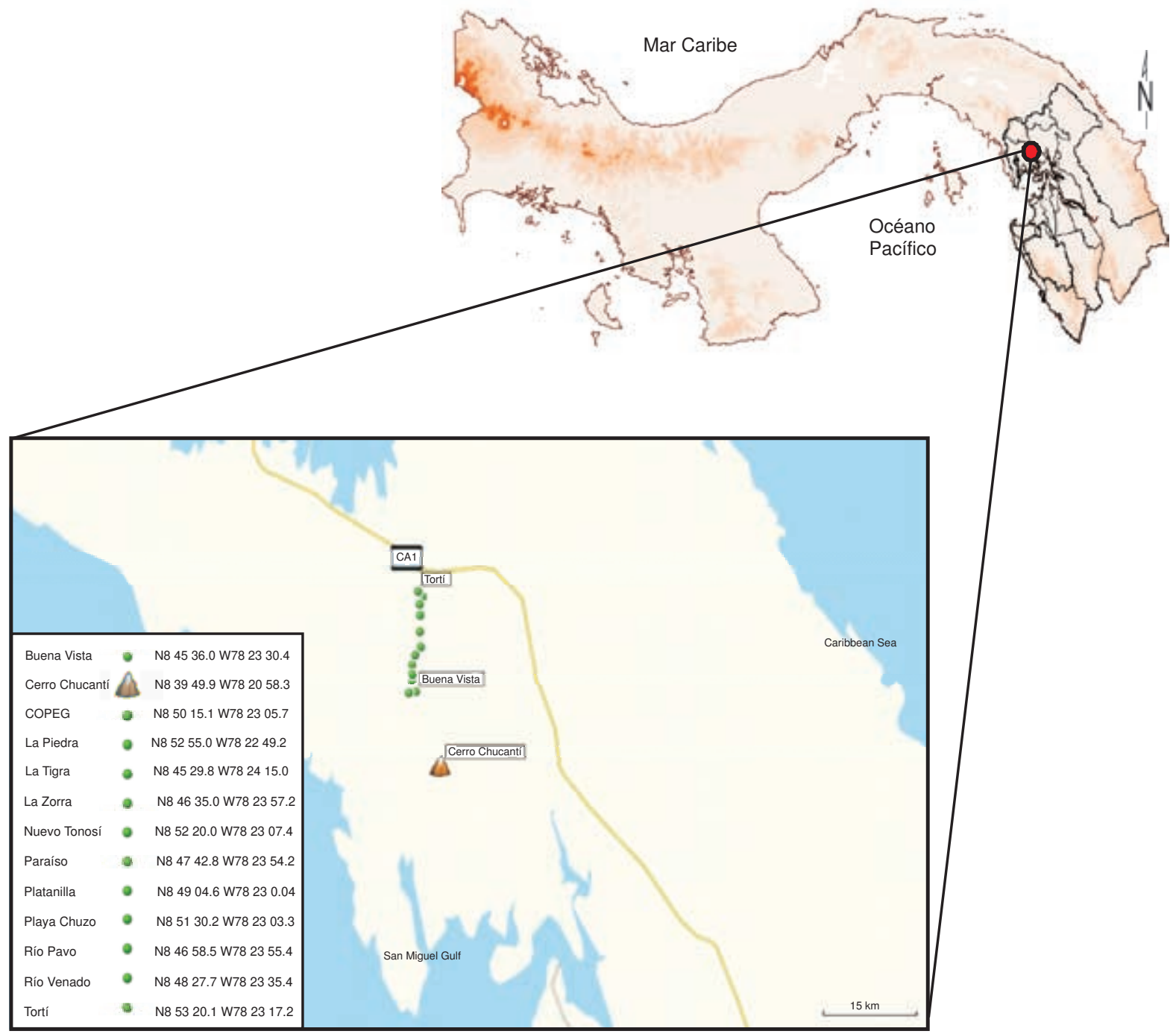

Figura 1. Localización de la Reserva Natural Privada Cerro Chucantí

trampas permanecieron en el campo de las 16:00 hasta las 07:00. Los mamíferos capturados se anestesiaron siguiendo los protocolos de la Organización Panamericana de la Salud (OPS). (http://www.paho.org/Spanish/AD/DPC/CD/ hct_98104-protocolo.pdf). Todos los ectoparásitos recolectados se preservaron en etanol al $95 \%$. Las garrapatas se identificaron utilizando la clave de Fairchild, et al. (14).

\section{Análisis de laboratorio}

Las pruebas moleculares se practicaron únicamente en garrapatas y pulgas, agrupando 3 a 7 pulgas 0 garrapatas inmaduras por cada especie o huésped en viales individuales. Las garrapatas machos y hembras no ingurgitadas se separaron en grupos de 1 a 2 individuos, mientras que las hembras ingurgitadas se procesaron individualmente. El material genético se extrajo por medio del kit de extracción de tejido DNeasy ${ }^{\mathrm{TM}}$ (Qiagen, Hilden, Alemania), siguiendo los criterios del fabricante.

Se utilizaron los iniciadores CS-78 y CS-323, que amplifican una porción de 401 pb del gen de la sintetasa de citrato ( $g l t A)$, según Labruna, et al. (15). A los amplicones con un tamaño esperado, se les hicieron nuevos análisis utilizando los iniciadores gltA (CS-239 y CS 1069) y ompA (RS 190.7 y RS 190.602) (15,16). Para comprobar el tamaño de las bandas resultantes, se utilizó electroforesis en gel de agarosa ( $1 \%$ ) en cada producto amplificado. Se utilizó material genético extraído de Rhipicephalus sp. como control positivo y, agua, como control negativo.

Para ambos genes, los amplicones que presentaban el tamaño esperado, se purificaron usando 
ExoSap $^{\mathrm{TM}}$ (USB) y se secuenciaron en un secuenciador automatizado (Applied Biosystem, Perkin Elmer, modelo ABI Prism 310 Genetic, California, EU). Para comparar con otras Rickettsia, las secuencias parciales obtenidas se alinearon con las reportadas en BLAST.

\section{Resultados}

Se examinaron 95 animales domésticos, correspondientes a 47 perros, 38 caballos, cinco gatos, tres vacas y dos ovejas. A éstos, se les extrajeron garrapatas Dermacentor nitens, Amblyomma cajennense s.I., Rhipicephalus sanguineus s.I., $R$. (Boophilus) microplus y pulgas Ctenocephalides felis. Amblyomma cajennense fue la única garrapata encontrada en pastizales, mientras que en los bosques se encontraron larvas y ninfas de una especie no identificada de Amblyomma. Ninfas y adultos de $A$. cajennense fueron las únicas garrapatas extraídas de humanos. El listado de ectoparásitos extraídos de mamíferos domésticos se detalla en el cuadro 1.

Se capturaron 48 individuos de mamíferos silvestres, los cuales se describen en el cuadro 2. No se encontraron ectoparásitos en Marmosiops impavidus, Dasypus novencinctus, Heteromys desmarestianus, Heteromys spp., Tylomys watsonii y Zygodontomys brevicauda.

Se realizaron 425 reacciones de PCR, resultando 270 negativas y 155 positivas. De las positivas, 86 amplificaron para el gen gltA (55\% de los positivas) y 41 (26\%) para ompA. Estas positivas correspondieron a 29 pools de $D$. nitens, 27 de $A$. cajennense, 14 de $R$. sanguineus y 16 de $C$. felis. Las secuencias de consenso de 1.053 pb del gen
gltA y $564 \mathrm{pb}$ de ompA, tuvieron un porcentaje de cobertura para $R$. amblyommii de 99,7 a $100 \%$ y 99,8 a $100 \%$, respectivamente. Este material genético fue hallado en 10 pools de $A$. cajennense, 6 de $D$. nitens, 4 de $R$. sanguineus y en 3 de ninfas de Amblyomma spp. Igualmente, se obtuvieron secuencias de consenso de $724 \mathrm{pb}$ del gen gltA con un porcentaje de cobertura para $R$. felis de 97,8 a $99,8 \%$. El material genético de $R$. felis se detectó en 10 pools de $C$. felis y uno de $A$. cajennense.

No se encontró ADN de Rickettsia spp. en las garrapatas Ixodes venezuelensis, $A$. ovale, $A$. oblongoguttatum, A. sabanerae o R. (B.) microplus, ni en las pulgas Rhopalopsyllus sp.

\section{Discusión}

En este trabajo se encontró que $R$. amblyommii fue la especie más comúnmente detectada en la zona de estudio, al haberse detectado ADN de esta especie en $A$. cajennense s.l., $D$. nitens, $R$. sanguineus s.l. y ninfas de Amblyomma spp. en fase de vida libre. El hallazgo de ADN en ninfas de vida libre, sugiere que las mismas mantuvieron la infección durante la muda de larva a ninfa; sin embargo, harían falta más estudios experimentales que sustenten este hecho.

La circulación en fauna doméstica puede deberse a las interacciones entre las distintas especies de animales domésticos (por ejemplo, caballos, bovinos y perros que cohabitan en un mismo sitio) o potenciadas por el número de garrapatas infectadas que pueden parasitarlos. En este trabajo no se obtuvieron amplificaciones de Rickettsia spp. en ectoparásitos de fauna silvestre, lo cual pudo deberse a los pocos individuos analizados, lo que

Cuadro 1. Especie de Rickettsia detectada según las especies de ectoparásitos extraídos y sus huéspedes

\begin{tabular}{|c|c|c|c|c|}
\hline \multirow[b]{2}{*}{ Huésped } & \multirow[b]{2}{*}{ Ectoparásitos } & \multirow[b]{2}{*}{$\begin{array}{c}\text { n-parasitados/ } \\
\text { n-total }\end{array}$} & \multicolumn{2}{|c|}{ Secuencia } \\
\hline & & & $\begin{array}{c}\text { Rickettsia } \\
\text { amblyommii }\end{array}$ & $\begin{array}{c}\text { Rickettsia } \\
\text { felis }\end{array}$ \\
\hline Gato & Ctenocephalides felis & $5 / 5$ & - & 2 \\
\hline \multirow[t]{2}{*}{ Vaca } & Amblyomma cajennense & $3 / 3$ & - & - \\
\hline & Rhipicephalus microplus & $3 / 3$ & - & - \\
\hline \multirow[t]{4}{*}{ Perro } & Ctenocephalides felis & $47 / 47$ & 9 & 6 \\
\hline & Amblyomma cajennense & $28 / 47$ & 7 & - \\
\hline & Amblyomma ovale & $31 / 47$ & - & - \\
\hline & Rhipicephalus sanguineus & $45 / 47$ & 4 & - \\
\hline \multirow[t]{2}{*}{ Caballo } & Amblyomma cajennense & $38 / 38$ & 2 & 1 \\
\hline & Dermacentor nitens & $38 / 38$ & 6 & - \\
\hline Carnero-oveja & Rhipicephalus microplus & $1 / 2$ & - & - \\
\hline Humano & Amblyomma spp. & $5 / 15$ & - & - \\
\hline en campo & Amblyomma spp. & & 1 & - \\
\hline
\end{tabular}


Cuadro 2. Ectoparásitos encontrados en animales silvestres en la Reserva Natural Privada Cerro Chucantí, Panamá

\begin{tabular}{|c|c|c|}
\hline Huésped & Ectoparásitos & Número de individuos \\
\hline \multicolumn{3}{|l|}{ Orden Testudinata } \\
\hline Kinosternum spp. & Amblyomma sabanerae & 3 machos \\
\hline \multicolumn{3}{|l|}{ Orden Marsupialis } \\
\hline \multicolumn{3}{|l|}{ Familia Didelphidae } \\
\hline \multirow[t]{3}{*}{ Didelphis marsupialis } & Amblyomma sp. & 13 larvas, 21 ninfas \\
\hline & Ctenocephalides felis & 19 machos y hembras \\
\hline & Rhopalopsyllus sp. & \\
\hline \multicolumn{3}{|l|}{ Orden Rodentia } \\
\hline \multicolumn{3}{|l|}{ Familia Cricetidae } \\
\hline Melanomys caliginosus & Amblyomma sp. & 2 larvas \\
\hline Oryzomys sp. & Ixodes venezuelensis & Una hembra \\
\hline \multicolumn{3}{|l|}{ Familia Cuniculidae } \\
\hline Agouti paca & Amblyomma sp. & 4 larvas \\
\hline \multicolumn{3}{|l|}{ Familia Echymidae } \\
\hline \multirow[t]{5}{*}{ Proechymis semispinosus } & Amblyomma sp. & 24 larvas, 35 ninfas \\
\hline & Harrisonia uncinata & 35 individuos \\
\hline & Gyropus sp. & 25 individuos \\
\hline & Gliricola sp. & 37 individuos \\
\hline & Rhopalopsyllus sp. & \\
\hline \multirow{2}{*}{\multicolumn{3}{|c|}{$\begin{array}{l}\text { Orden Carnivora } \\
\quad \text { Familia Canidae }\end{array}$}} \\
\hline & & \\
\hline \multirow[t]{4}{*}{ Cerdocyon thous } & Amblyomma oblongoguttatum & Dos machos \\
\hline & & Un macho y una hembra \\
\hline & Amblyomma ovale & \\
\hline & Rhipicephalus sanguineus & Dos machos \\
\hline
\end{tabular}

hace posible que mayores muestras provean más información.

En Panamá, otras investigaciones han reportado la presencia de ADN de $R$. amblyommii en $A$. cajennense, $D$. nitens, $R$. sanguineus y en $A$. ovale $(11,12)$. De éstas, $A$. cajennense es la más común en ambientes perturbados de Panamá (14), además de considerarse la garrapata de mayor relevancia en salud pública, tanto por el número de patógenos que pudiera transmitir, como por ser una de las especies que más parasita humanos $(17,18)$.

En otras zonas del neotrópico, $R$. amblyommii se ha detectado en $A$. coelebs, $A$. cajennense $(18,19)$ y $A$. neumanni (20). Según Labruna (5), esta especie, al igual que $R$. bellii, mantiene una tasa de infección en garrapatas que varía entre 10 y $100 \%$, siendo probable que no sean patógenas para las garrapatas, lo cual la diferenciaría de $R$. rickettsii. La alta tasa de supervivencia de las garrapatas $R$. amblyommii, podría influir en la capacidad de determinadas especies, y sus estadios, para transmitir la bacteria. Este señalamiento parece ser corroborado por Williamson, et al. (21), quienes expresan que el parasitismo en masa de larvas no ingurgitadas de $A$. americanum puede aumentar la posibilidad de infección.
En vertebrados, incluidos los humanos, la capacidad patógena de R. amblyommii es discutida (22). Los datos serológicos sugieren que esta especie puede causar cuadros febriles leves en pacientes, además de ser el agente responsable de una alta tasa de seropositivos en zonas infectadas con garrapatas del género Amblyomma (23-25).

En animales domésticos se ha reportado serorreacción a antígenos contra $R$. amblyommii especialmente en suero de perros de Brasil (29) y en suero de perros y caballos de Panamá (12). Estos datos parecen sugerir que los perros y caballos pueden mantener infecciones peridomésticas de $R$. amblyommii. Además, los hallazgos en garrapatas como $A$. coelebs, indican la posibilidad de que animales silvestres estén infectados con esta especie, haciendo necesarios mayores estudios en garrapatas y mamíferos silvestres.

Con respecto a $R$. felis, esta especie se ha reportado en 20 países alrededor de los cinco continentes (26). Se considera que la pulga de perros y gatos, $C$. felis, es vector biológico de $R$. felis $(26,27)$, y se calcula que un alto porcentaje de estas pulgas está infectada con esta especie (5). Aparte de $C$. felis, $R$. felis se ha detectado en, al menos, otras 12 especies de pulgas, además de 
ácaros Astigmata, Mesostigmata, varias especies de garrapatas $(27,28)$ y en los piojos de libros Liposcelis bostrychophila (29). Hasta el momento, los hallazgos serológicos sugieren que la zarigüeya Didelphis virgiana y los gatos pueden ser infectados por R. felis (30-32).

Según algunas investigaciones de laboratorio, $R$. felis puede mantener infecciones en pulgas por, al menos, 12 generaciones, sin la necesidad de la ingestión de sangre infectada, lo que demuestra su capacidad de transmitirse verticalmente (30). Esto, sumado a la proximidad de $C$. felis con mascotas (perros y gatos), haría que una gran parte de la población humana esté expuesta a infecciones por $R$. felis. Según Parola (26), alrededor del mundo se han reportado cerca de 100 registros de rickettsiosis provocadas por $R$. felis en humanos, siendo posible que existan otros casos no diagnosticados adecuadamente.

\section{Agradecimiento}

A la Autoridad Nacional del Ambiente por los permisos de captura. De la misma manera, se agradece a Guido Berguido y los trabajadores de Panama Advantage Tour, por el transporte y guía en Cerro Chucantí, especialmente a Jesús, Orlando, Víctor y Euclides. Finalmente, se agradece a Vilma Fernández, José Montenegro, Mileyka Santos y Eduardo Santamaría, por su apoyo en la colocación de trampas y manipulación de los mamíferos capturados.

\section{Conflicto de intereses}

El manuscrito fue preparado y revisado con la participación de todos los autores, quienes declaramos que no existe ningún conflicto de intereses que ponga en riesgo la validez de los resultados.

\section{Financiación}

El proyecto fue auspiciado por la Secretaría Nacional de Ciencia y Tecnología (SENACYT) mediante la acreditación al fondo COL-07-045.

\section{Referencias}

1. Szabó MP, Labruna MB, Pereira M, Duarte J. Ticks (Acari: Ixodidae) on wild marsch-deer (Blastocerus dichotomus) from Southeast Brazil: Infestations before and after habitat loss. J Med Entomol. 2003;40:268-74.

2. Pereira CM, Szabo MP, Bechara GH, Matushima ER, Duarte JM, Rechav Y, et al. Ticks (Acari: Ixodidae) associated with wild animals in the Pantanal region of Brazil. J Med Entomol. 2000;37:979-83.

3. Fournier P, Raoult D. Current knowledge on phylogeny and taxonomy of Rickettsia spp. Ann N Y Acad Sci. 2009; 1166:1-11.
4. Estripeaut $D$, Aramburú $M$, Saéz-Llórens $X$, Thompson H, Dasch G, Paddock C, et al. Rocky Mountain spotted fever, Panama. Emerg Infect Dis. 2007;13:1763-5.

5. Labruna M. Ecology of Rickettsia on South America. Ann N Y Acad Sci. 2009;1166:156-66.

6. Calero C. Outbreak of the Thypus of the murine type. First record from the Isthmus of Panama. Am J Trop Med. 1948;28:313-21.

7. Rodaniche E. Natural infection of the tick, Amblyomma cajennenses, with Rickettsia rickettsii in Panama. Am J Trop Med Hyg. 1953;2:696-9.

8. Rodaniche EC, Rodaniche A. Spotted fever in Panama; isolation of the etiologic agent from a fatal case. Am J Trop Med Hyg. 1950;30:511-7.

9. Yunker CE, Brennan JM, Hughes LE, Philip CB, Clifford $\mathrm{CM}$, Peralta $\mathrm{PH}$, et al. Isolation of viral and rickettsial agents from Panamanian Acarina. J Med Entomol. 1975;12:250-5.

10. Eremeeva M, Karpathy S, Levin M, Caballero M, Bermúdez SE, Dasch G, et al. Spotted fever rickettsiae, Ehrlichia and Anaplasma, in ticks from peridomestic environments in Panamá. Clin Microbiol Infec Dis. 2009;15(Suppl.2):2-3.

11. Bermúdez SE, Eremeeva ME, Karpathy SE, Samudio FE, Zambrano ML, Zaldívar YL, etal. Detection and identification of rickettsial agents in ticks from domestic mammals in Eastern Panama. J Med Entomol. 2009;46:856-61.

12. Bermúdez SE, Zaldívar Y, Spolidorio M, Moraes-Filho $\mathbf{J}$, Miranda R, Caballero $\mathbf{C}$, et al. Rickettsial infection in domestic mammals and their ectoparasites in El Valle de Antón, Coclé, Panamá. Vet Parasitol. 2011;177:134-8.

13. Aizprúa R. Primer acercamiento a la flora de la Estación Biológica de Chucantí, Darién. 2006. Fecha de consulta: 14 de mayo de 2011. Disponible en: http://advantagepanama. com/chucanti

14. Fairchild G, Kohls G, Tipton V. The ticks of Panamá. En: Wenzel RL, Tipton VJ, editores. Ectoparasites of Panamá. Chicago: Field Museum of Natural History; 1966. p. 167219

15. Labruna MB, Whitworth T, Horta MC, Bouyer DH, McBride JW, Pinter A, et al. Rickettsia species infecting Amblyomma cooperi ticks from an area in the state of São Paulo, Brazil, where Brazilian spotted fever is endemic. J Clin Microbiol. 2004;42:90-8.

16. Labruna MB, Whitworth T, Bouyer DH, McBride JW, Camargo LM, Camargo EP, et al. Rickettsia bellii and Rickettsia amblyommii in Amblyomma ticks from the state of Rondônia, Western Amazon, Brazil. J Med Entomol. 2004;41:1073-81

17. Guglielmone AA, Estrada-Peña A, Keirans J, Robbins R. Las garrapatas (Acari: Ixodida) de la región zoogeográfica neotropical. Buenos Aires: Instituto Nacional de Tecnología Agropecuaria; 2004. p. 142.

18. Estrada-Peña A, Guglielmone AA, Mangold AJ. The distribution and ecological "preferences" of the tick Amblyomma cajennense (Acari: Ixodidae), an ectoparasite of humans and other mammals in the Americas. Ann Trop Med Parasitol. 2004:98:283-92.

19. Parola DP, Matsumoto K, Socolovschi C, Parzy D, Raoult D. A tick-borne rickettsia of the spotted-fever group, similar 
to Rickettsia amblyommii, in French Guyana. Ann Trop Med Parasitol. 2007;101:185-8.

20. Labruna MB, Pacheco RC, Nava S, Brandao PE, Richtzenhain LJ, Guglielmone AA. Infection by Rickettsia belliiand Candidatus "Rickettsia amblyommil" in Amblyomma Neumanni ticks from Argentina. Microb Ecol. 2007;54:12633.

21. Williamson P, Billingley P, Teltow G, Seals J, Turnbough, Atkinson S. Borrelia, Ehrlichia, and Rickettsia spp. in ticks removed from persons, Texas, USA. Emerg Infec Dis. 2009;16:441-6.

22. Mediannikov O, Paddock C, Parola P. Other rickettsiae of possible or undetermined pathogenicity. Ann N Y Acad Sci. 2009;1166:163-77.

23. Dasch G, D Kelly, A Richards J, Sánchez, Rives C. Western blotting analysis of sera from military personnel exhibiting serological reactivity to spotted fever group rickettsiae. Am J Trop Med Hyg. 1993;49:220.

24. Parola P, Paddock C, Raoult D. Tick borne rickettsiosis around the world: Emerging diseases challenging old concepts. Clin Microbiol Rev. 2005;18:719-56.

25. Apperson C, Engber B, Nicholson W, Mead D, Engel J, Yabsley M, et al. Tick-borne diseases in North Carolina: Is "Rickettsia amblyommir" a possible cause of rickettsiosis reported as Rocky Mountain spotted fever? Vector Borne Zoonotic Dis. 2008;8:597-606.

26. Parola P. Rickettsia felis from a rare disease in the USA to a common cause of fever in sub-Saharan Africa. Clin Microbiol Infect. 2011;17:996-1000.

27. Reif K, Macaluso KR. Ecology of Rickettsia felis: A review. J Med Entomol. 2009;46:723-36.

28. Horta M, Labruna M, Pinter A, Linardi PM, Schumaker T. Rickettsia infection in five areas of the state of Sao Paulo, Brazil. Mem Inst Oswaldo Cruz. 2007;102:793-801.

29. Behar A, McCormick LJ, Perlman SJ. Rickettsia felis infection in a common household insect pest, Liposcelis bostrychophila (Psocoptera: Liposcelidae). Appl Environ Microbiol. 2010;76:2280-5.

30. Wedincamp J, Foil LD. Vertical transmission of Rickettsia felis in the cat flea (Ctenocephalides felis Bouche). J Vector Ecol. 2002;27:96-101.

31. Boostrom A, Beier MS, Macaluso JA, Macaluso KR, Sprenger D, Hayes J, et al. Geographic association of Rickettsia felis-infected opossums with human murine typhus, Texas. Emerg Infect Dis 2002;8:549-54.

32. Labruna M, Ogrzewalska M, Moraes-Filho J, Lepe P, Gallegos JL, López J. Rickettsia felis in Chile. Emerg Infect Dis. 2007;13:1794-5. 